\section{DETAILS IN TWO COMMONLY USED URINARY TESTS}

\author{
F. H. CHURCH, A.M., M.D. \\ UTICA, N. Y.
}

Those using the Jaffé test for the identification of indican or the indoxyl sulphates in the urine frequently meet an equivocal result. Instead of the expected indigoblue-colored chloroform solution, the solvent takes on a recldish or reddish-violet tint. 'This may be due either to indigo-red or, more commonly, to the presence of iodicls in the urine. In the latter case, if the iodid is present in large amounts, it may be identified by adding starch water and acidifying directly. If the iodid is present only in small amounts, the chloroform should be removed with the pipette and placed in another testtube. A little starch water is added and the material made alkaline by the addition of a little sodium or potassium hydroxid. The iodid of the alkali is formed and goes into solution in the starch water. Now on the addition of a little acid the iodin is set free with the characteristic starch-iodin reaction.

According to the customary directions in the textbooks for the performance of the Gerhardt test for acetoacetic acid, on the addition of the ferric chlorid solution to the urine a marked and obscuring precipitate of phosphates is usually obtained. This may be avoided and much time and temper saved by the chemist's simple expedient of adding the urine drop by drop to 10 or 15 c.c. of the ferric chlorid solution. In this way it is also possible to get a rough idea of the amount of the acetoacetic acid present. If this is present in quantities it will give a strong Bordeaux red coloration with the addition of a very few drops of the urine; otherwise this coloration will not be present until 1 or 2 c.c. of the urine have been added.

Furthermore, it is possible to vary the strength of the reaction by varying the acidity of the ferric chlorid. After the reaction is performed and in the presence of a weak or indeterminate result, the addition of a very little dilute acid or alkali will have a tendency to accentuate the color. The material may then be halved and one tube boiled. The diacetic acid is, of course, broken up and the red reaction clears up just as it would on standing for twenty-four or forty-eight hours. These facts hold for the behavior of the diacetate of ethyl in aqueous solution as well as the acid or more probably its esters in the urine.

43 Gardner Building.

Professional Secrecy and Accident Insurance.-An article in the Presse Médicale, April 17, by a professor in the law department of the university at Montpellier and a member of the medical faculty, discusses this subject and suggests that in making out a certificate in regard to an accident, it should be handed when completed to the patient himself or his representatives. By not giving the certificate to any one but the victim of the accident, the physician is relieved from the risk of breach of the professional obligation to secrecy. The certificate should be read to the victim or his representatives and the technical terms explained to them so that they can not plead ignorance of their meaning later. Unless objective signs of a pathologic taint, not affecting the trauma, are discovered, it is unnecessary to certify to it, and the patient's statements in regard to a pathologic past for which no objective findings can be detected should be regarded as confidential. The discussion was suggested by a recent case in which a workman sued the physician for $\$ 400$ damages because in his certificate he mentioned the presence of gonococeal urethritis. The case was non-suited finally.

\section{TORSION OF PEDICLE OF AN OVARIAN} CYSTOMA IN A YOUNG GIRL

\section{BORNSTEIN, M.D. \\ MILWAUKEE, WIS.}

A number of cases of ovarian cysts with twisted pedicles in girls below the age of fourteen are to be found in medical literature. Nevertheless, there is a common liability of overlooking this condition and in differentiating it from the more conmon inflammatory conditions in the lower abdomen. The following case should be of interest:

History--B. S., female, aged 13; had never menstruated, but for three months past had complained of perioclic attalckis of pain, usually at the end of each month, starting in the right knee, appearing in the thigh and groin of the same side. Right hip-joint was normal. A physician diagnosed the first attack one of appendicitis but operation was refused. On the evening of March 31 she had a similar attack with a sharp pain in the right iliac region; developed fever and romitel. She was examined next morning, but her physician could find no local tenderness on palpation. The abdomen was distended and vomiting continued; the pulse became rapid and feeble.

Operation.-She was admitted to Mount Sinai Hospital on the evening of March 31 and operated on in the clinic of Dr. H. Greenberg. Abdomen was opened over the right iliac fossa. A local peritonitis was present. The appendix appeared normal but contained several dried-up concretions. Palpation disclosed a large cystic mass springing from the right side of the pelvis. It was found to be a large ovarian cyst, about the size of a man's head, with its pedicle completely twisted. The cyst was emptied and removed. Its contents were fluid and straw colored; the wall bluish and contained several yellowish, thickened masses. The appendix was removed and the abdomen closed.

During the same month Dr. Greenberg operated on a young woman (24 years old) for the same trouble. She was brought in with a diagnosis of acute appendicitis. Incision disclosed a blackened ovarian cyst with a completely twisted pedicle. Both patients have been discharged, cured.

733 Central Avenue.

\section{Therapeutics}

\section{THE PREVENTION OF CONTAGION}

Perhaps the most valuable service a physician renders is the prevention of the spread of contagions discase in the family of his patient, and the prevention of the sprear of disease in the communitr. While Boards of Health assume the responsibility of preventing the spread of the contagion, it is only in exceptional instances that they can assume the whole responsilitity, and if the physician who has charge of the patient is not efficient in his sanitary measures, the advice, orders and restrictions of the Board of Health become almost valueless. Therefore, it is not only the highest aim, but it is the duty of the attending physician to isolate the patient and to prevent the spread of the contagium that others shall not acquire the disease.

To prevent contagion properly, the most important prerequisite is that the physician shal understand the means by which the contagium of each particular disease is spread. Dr. A. H. Doty, Health Officer of the Port of New York, in the American Jommal of the Medical Sciences, July, 1909, discusses this subject, and his long years of experience plus his efficiency as health officer makes his advice and his scientific conclusions most valuable.

Yellow fever is transmitted by the mosquito only, and clothing, bedding, and even the excretions of the 